\title{
The Motion Planning of Overhead Crane Based on Suppressing Payload Residual Swing
}

\author{
Hua-sen LIU ${ }^{1}$, Wen-min CHENG $^{1}$ \\ ${ }^{1}$ Southwest Jiaotong University, Research Institute of Mechanical Engineering, 610031 Chengdu, China
}

\begin{abstract}
Since the overhead crane system is subject to under actuation system due to that overhead crane and payload are connected by flexibility wire rope. The payload generates residual swing when the overhead crane is accelerating/ decelerating the motions. This may cause trouble for the payload precise positioning and motion planning. Hence, an optimization input shaping control method is presented to reduce the under actuated overhead crane's payload swing caused via the inertia force. The dynamic model of the overhead crane is proposed according to the physics structure of the crane. The input shaper based on the motion planning of the crane is used as the feed forward input to suppress payload residual swing. Simulation and experiment results indicate that the ZV input shaper and ZVD input shaper can reduce the payload swing of the overhead crane.
\end{abstract}

\section{Introduction}

The payload of the overhead crane belongs to a under actuated system so that the payload swings when there are inertial forces acted on the payload. The residual swing of payload wastes a lot of time and may cause crash between the payload and the installation parts for the high precision installation. On the other hand, the residual swings of the payload cause trouble for the payload precise positioning and motion planning. The higher payloads handling rates requires the motion planning of overhead crane with the payload residual swing suppressed.

Many research groups [1] have done their great effect for the motion planning of overhead crane. A synopsis of crane system dynamics [2] that incorporates the effects of nonlinear drives and motors was derived. A combined feedback and input shaping controller was developed to address both sources of oscillation. The quay container cranes [3] was modelled by software ADMAS and the simulation results were verified by the experiments, which was an efficiently method compute dynamic responses by the virtual prototype technology. In Ref.[4], the payload swing caused by base excitation was investigated and limited by using reeling and unreeling the hoisting cable. In order to suppress the payload residual swing, the resultant crane control schemes developed include nonlinear controls [5], sliding-mode controls, fuzzy controls, input shaper controls and image control. Payload oscillations have a significant impact on the stability of cranes. Lee et al. [6] proposed a slidingmode anti-swing control for overhead cranes to realize an anti-swing trajectory control with high-speed load hoisting. Lee [7] proposed a motion-planning method based on the Lyapunov stability theorem for a highperformance anti-swing control of overhead cranes. Kuo [8] used two related acceleration and deceleration to propose a three-stagemethod consisting of piecewise acceleration, constant speed, and piecewise deceleration. The proposed method maintained a small sway angle in fast speed. Bartolini [9] proposed a simple control scheme, based on second-order sliding modes, which guarantees a fast and precise load transfer and the swing suppression during the load movement, despite of model uncertainties and unmodeled dynamic actuators. A physics-based simulation and animation of crane motions [10] was also developed to visualize vibrations induced by crane motion.

The following section establish a mathematical model of the overhead crane's payload according to the physics structure of the crane. Based on the swing frequency of the payload, section 3 designs the input shaping controller for the motion planning of overhead crane payload control system. In section 4, numerical simulations and experimental are contrasted to vivificate the effective of the motion planning for overhead cranes.

\section{Mathematical Model of an Overhead Crane}

In this section, a dynamic model for the overhead crane is derived which is shown in figure $1 . O$-xy is the trolley coordinate system which motions with the trolley. The 
axes of the crane coordinate frame are chosen to coincide with the principal axes of the crane payload. Also, the origin $O$ is located at the center gravity of the crane's trolley. The trolley motions along the girder in the $\mathrm{x}$ (traverses) direction. The origin of the trolley coordinate system is $(\mathrm{x}, \mathrm{y})$ in the fixed coordinate system. The payload is hoisted from the trolley by wire rope. The payload swing around the point $O$ with an angle $\theta$. The trolley is considered as point masses with a mass of $m_{1}$, and the mass of the payload is $m_{2}$ which also considered as a point mass. The rope is assumed to be non-elastic and fixed length $l$.

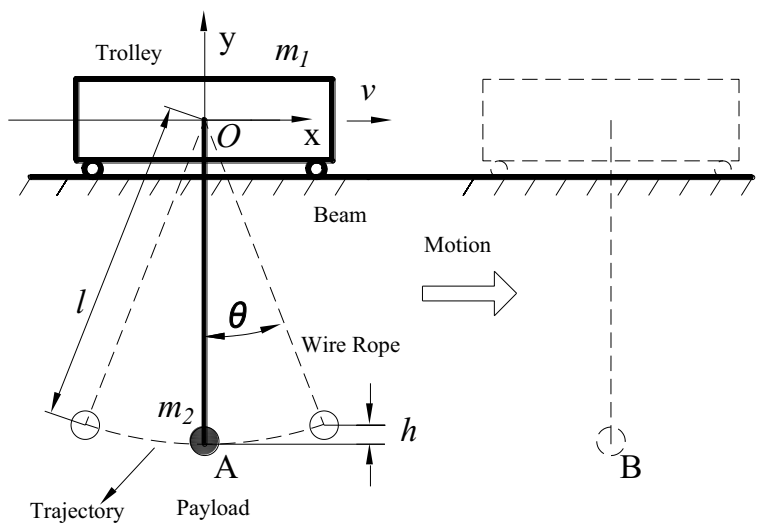

Figure 1. Dynamic model of overhead crane

The position vector of the center of gravity of payload $\left(x_{p}, y_{p}\right)$ in the fixed coordinate system is:

$$
\left\{\begin{array}{l}
x_{p}=x+l \sin \theta \\
y_{p}=-l \cos \theta
\end{array}\right.
$$

The kinetic energy of the overhead crane system $T$ is:

$$
T=\frac{1}{2} m_{1} \dot{x}^{2}+\frac{1}{2} m_{2}\left(\dot{x}_{p}^{2}+\dot{y}_{p}^{2}\right)
$$

where $v$ denotes the velocity of the crane's trolley.

Using the center of gravity of trolley as the zero potential energy, the total potential energy $(U)$ of the overhead crane system is:

$$
U=-m_{2} g l \cos \theta
$$

Based on the Lagrange dynamic equation, the dynamic equations of an overhead crane system can be expressed by:

$$
\frac{\mathrm{d}}{\mathrm{d} t}\left(\frac{\partial L}{\partial \dot{q}}\right)-\frac{\partial L}{\partial q}=Q
$$

where $L=T-U, q=[x, \theta]^{T}$ are the generalized displacements, including the trolley motion along the girder $\mathrm{x}$, the payload swing in the two directions: $\theta . Q=\left[F_{x}, F_{\theta}\right]^{T}$ are the generalized forces, including the force $F_{x}$ acted on the trolley along with the girder, the air friction acted on the payload $F_{\theta}$.

The equations of motion of the crane system are obtained by inserting $L$ and $Q$ into Lagrange's equation associated with the generalized coordinates $x, \theta$. As the swing angles of payload are small, so $\sin \theta \approx \theta, \quad \cos \theta \approx 1$, and the high order terms in the nonlinear model can be neglected. Simplify the Eq.(7) as the three following equations:

$$
\begin{gathered}
\left(m_{1}+m_{2}\right) \ddot{x}+m_{2} l \ddot{\theta}=F_{x} \\
m_{2} l(\ddot{x}+l \ddot{\theta}+\mathrm{g} \theta)=F_{\theta}
\end{gathered}
$$

When the overhead crane loads the payload, the payload swings along with the crane's trolley direction (the $\mathrm{x}$ direction in figure 1). The payload and the crane's trolley compose a complex coupling system. The dynamic differential Equations of the overhead crane are given by Eq. (5) (6). There is a driving force or braking force $F_{x}$ in horizontal acted on the trolley. There are air friction as well as reel friction force $F_{\theta}$ in $\mathrm{x}$ direction acted on the payload, which is proportional to the angular velocity. Suppose that, $F_{\theta}=-\mu \dot{\theta}, \mu$ is a constant which represents the damping factor. Substituting equation (6) into equation (5), yields:

$$
\begin{aligned}
& \ddot{\theta}+\frac{1}{m_{1} m_{2} l^{2}}\left(m_{1}+m_{2}\right) \mu \dot{\theta} \\
& +\frac{1}{m_{1} l}\left(m_{1}+m_{2}\right) \mathrm{g} \theta=-\frac{F_{x}}{m_{1} l}
\end{aligned}
$$

The initial conditions are: $\theta(0)=\theta_{10}, \dot{\theta}(0)=v_{1}$. The general solution differential Eq. (7) is

$$
\theta(t)=A e^{-n t} \sin (\omega t+\varphi)+c
$$

$$
\begin{aligned}
& \text { Where: } n=\frac{\mu\left(m_{1}+m_{2}\right)}{2 m_{1} m_{2} l^{2}}, \quad k^{2}=\frac{\left(m_{1}+m_{2}\right)}{m_{1}} \frac{g}{l}, \\
& c=-\frac{F_{x}}{m_{1} l}, A=\sqrt{\left(\theta_{10}-c\right)^{2}+\frac{\left(v+n\left(\theta_{10}-c\right)\right)^{2}}{\omega^{2}}} \\
& \varphi=\arctan \left(\frac{\left(\theta_{10}-c\right) \omega}{v+n\left(\theta_{10}-c\right)}\right) \\
& \omega=\sqrt{\frac{\left(m_{1}+m_{2}\right)}{m_{1}} \frac{g}{l}-\frac{\mu^{2}\left(m_{1}+m_{2}\right)^{2}}{4 m_{1}^{2} m_{2}^{2} l^{4}}}
\end{aligned}
$$

\section{The Input Shaper for Motion Planning}

Input shaping is one of the wildly used methods to reduce the motion-induced swing, which is very easy to control and costs low. The input shaper is a powerful method to reduce the residual vibration of the payload, expressly when the trolley acceleration or deceleration. The parameters of the input shaper also are dependent on the distance of the payload.

\subsection{The response of Input Shaping}

The input amplitude and the input time are influenced by the frequency and the damping ratio. For an overhead crane system, the damping ratio is a constant. The typical of the two impulses ZV shaper's parameter is: 


$$
\left[\begin{array}{c}
V_{i} \\
t_{i}
\end{array}\right]=\left[\begin{array}{cc}
\frac{1}{1+K} & \frac{K}{1+K} \\
0 & 0.5 T_{d}
\end{array}\right](i=1,2)
$$

where the input amplitude is $V_{i}$ when the input time is $t_{i} . K$ is a parameter of the input amplitude, $T_{d}$ is the damped period of vibration,. $\omega_{d}$ is the damped vibration frequency, $\omega$ is the natural frequency, $\omega_{d}=\omega \sqrt{1-\zeta^{2}} \cdot \zeta$ is the damping ratio. $K=e^{\left(\frac{-\zeta \pi}{\sqrt{1-\zeta^{2}}}\right)}, T_{d}=\frac{2 \pi}{\omega_{d}}$

$$
\begin{aligned}
{\left[\begin{array}{l}
V_{i} \\
t_{i}
\end{array}\right] } & =\left[\begin{array}{cc}
\frac{1}{(1+K)^{2}} & \frac{2 K}{(1+K)^{2}} \\
0 & 0.5 T_{d}
\end{array}\right. \\
\text { Where } K=e^{\left(\frac{-\zeta \pi}{\sqrt{1-\zeta^{2}}}\right)}, T_{d} & =\frac{2 \pi}{\omega_{d}}
\end{aligned}
$$

A linear, vibratory system of any order can be specified as a cascaded set of second-order poles with the
ZV shaper's parameters are influenced by the system's frequency and damping ratio. However, the ZV shaper has a poor robustness for the system's frequency. When the system's frequency changing by disturbance, the effect of the reducing the residual swing by $\mathrm{ZV}$ shaper become weaken. In order to improve the frequency robustness of the $\mathrm{ZV}$ shaper, the partial derivative for the frequency of the $\mathrm{ZV}$ shaper sets to zero. The following is the typical of the three impulses ZVD shaper when the frequency of the system is $\omega_{d}$.

$$
y(t)=\left[V \frac{\omega}{\sqrt{1-\zeta^{2}}} e^{-\zeta \omega\left(t-t_{0}\right)}\right] \sin \left(\omega \sqrt{1-\zeta^{2}}\left(t-t_{0}\right)\right)
$$

Where $\mathrm{A}$ is the amplitude of the input impulse, $\omega$ is the undamped natural frequency of the plant, $\zeta$ is the damping ratio of the plant, $t$ is time, and $t_{0}$ is the time of the impulse input.

The Eq.(12) describes a step impulse response of the second-order underdamped system. For multiple impulse

$$
V(\zeta, \omega)=\frac{\omega}{\sqrt{1-\zeta^{2}}} \sqrt{\left[\sum_{i=1}^{n} V_{i} e^{-\zeta \omega\left(t-t_{i}\right)} \cos \left(\omega_{d}\left(t-t_{i}\right)\right)\right]^{2}+\left[\sum_{i=1}^{n} V_{i} e^{-\zeta \omega\left(t-t_{i}\right)} \sin \left(\omega_{d}\left(t-t_{i}\right)\right)\right]^{2}}
$$

inputs, such as $Z V$ input shaper, the system response can be obtained by the Vector Diagrams. When the impulses are composed by arbitrary inputs, the input response of the second order damped vibration system is:

Where, $\omega$ is the undamped vibration frequency and $\omega_{d}$ is the damped vibration frequency. $\omega_{d}=\omega \sqrt{1-\zeta^{2}}$.

$V(\zeta, \omega)$ is the system response vibration function, which is effected by the system nature frequency $\omega$ and the input shaper parameters $\zeta$.

\subsection{The Motion Planning Control}

Input shaping is a kind of method that convolution by the input signals and the impulse sequence. One of the advantages of the input shaping is that this method doesn't need the accuracy dynamic model of the system, it only need the frequency and the damping ratio of the system. Taking a ZV input shaper as an example as shown in Figure 2, the input velocity of the trolley is $V_{1}$ when the input time is $t_{1}$, and the input velocity of the trolley is $V_{2}$ when the input time is $t_{2}$. This is similar when the trolley decelerated motion. The region of $\Delta t_{1}$ is the acceleration motion stage of the trolley, and the region of $\Delta t_{3}$ is the deceleration motion stage of the trolley. Those two motion stages are designed by the input shaping to supress the residual swing of the payload. The region of $\Delta t_{2}$ is the uniform motion stage of the trolley.

In order to precise positioning for the payload, the displacement of the payload is a constant. The trolley's displacement $D$ from point $\mathrm{A}$ to point $\mathrm{B}$ is:

$$
D=V_{1} \bullet\left(\Delta t_{1}+\Delta t_{3}\right)+V_{2} \bullet \Delta t_{2}
$$



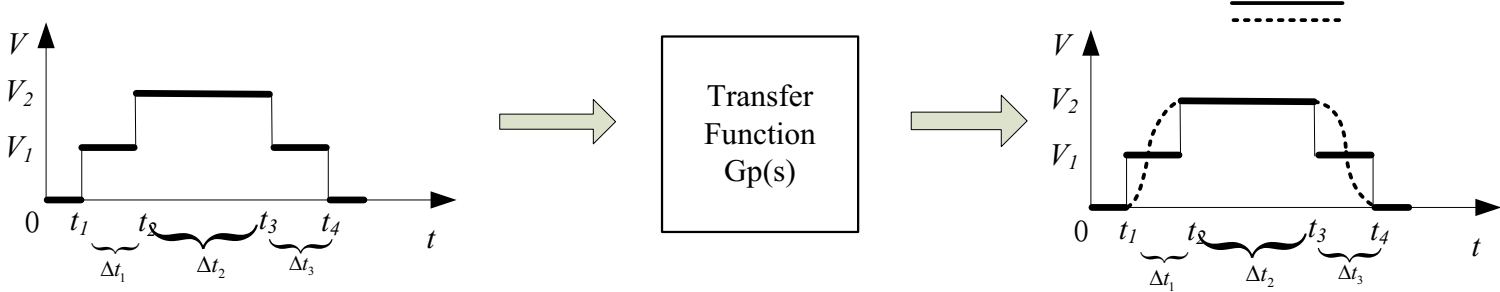

Figure 2. Schematic Diagram of Input Shaping

\section{Numerical Simulations and Experimental Validation}

The parameters of the input shaper can be solved by the constraint conditions of Eq. (9) (14). Taking a gantry container crane as an example to investigate the motion planning of payload. The gravitational acceleration is $g \approx 9.8 \mathrm{~m} / \mathrm{s}^{2}$, the mass of trolley is $m_{1}=10 \times 10^{3} \mathrm{~kg}$, the mass of payload is range $m_{2}=20 \times 10^{3} \mathrm{~kg}$, the length of wire cable is range from $l=10 \mathrm{~m}$.

\subsection{The Numerical Simulations of the Input Shapers}

The payload swing frequency is $1.7 \mathrm{rad} / \mathrm{s}$ according to the Eq.(9), the response of the payload residual swing amplitudes are shown in figure3. All of the payload residual swing amplitudes are near zero when the payload swing frequency is about $1.7 \mathrm{rad} / \mathrm{s}$. But if the input shaper frequency is not equal to the payload swing frequency, the robust of input shaper become weaker.

Figure 4 shows the input velocity of the trolley for $\mathrm{ZV}$ input shaper and ZVD input shaper. The ZV shaper has two input impulse sequence, and the ZVD input shaper has three input impulse sequence. The input amplitude and the input time of the two input shapers are difference. But the sum of the input amplitudes of two input shapers are equal. The input time of the ZVD shaper is about two times of that of ZV shaper.

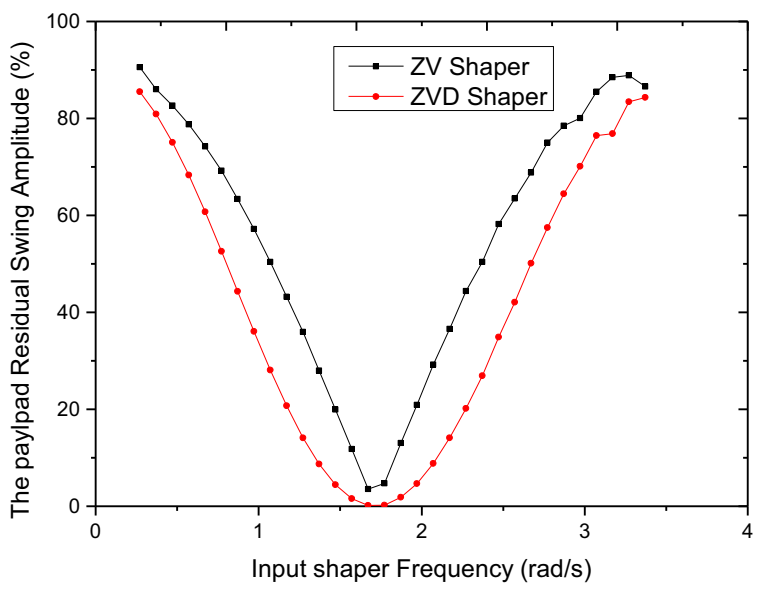

Figure3. The payload swing of three input shapers

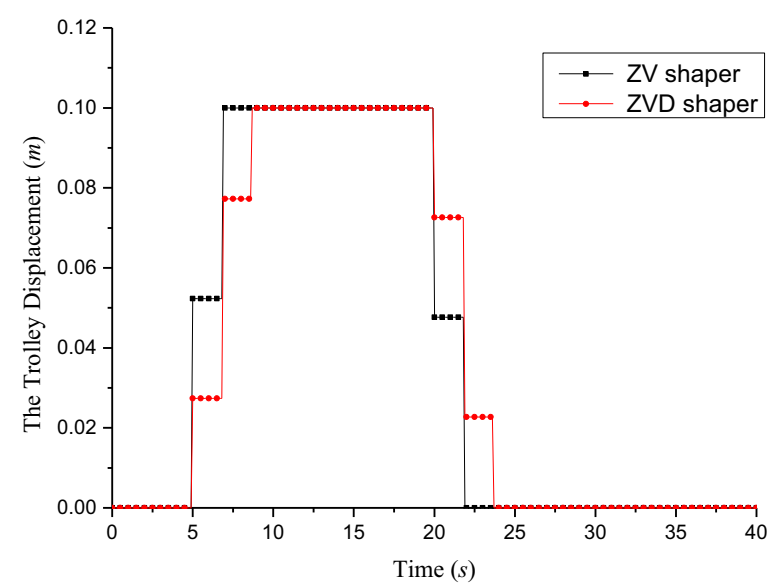

Figure 4. The input velocity of the trolley

The swing of the payload are shown in figure 5. The maximum payload swing of the ZV input shaper is about twice of that of ZVD input shaper. The residual payload swing after the time 25 seconds are similar.

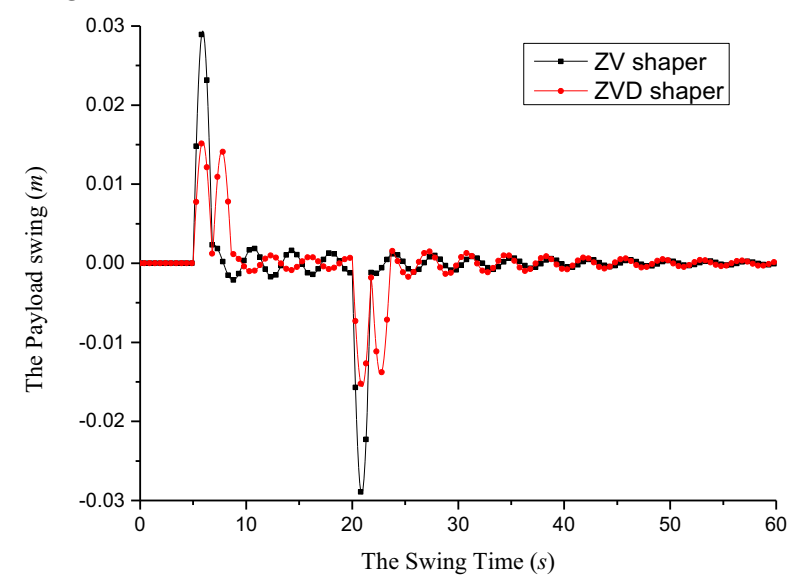

Figure 5. The swing of the payload

\subsection{The experimental validation}

To verify the residual swing of the payload, experimental model were performed on an overhead crane with single pendulum payload, which correspond to single mode input shaper and two-mode input shaper, respectively. A scaled model of an overhead crane with a maximum velocity of $0.1 \mathrm{~m} / \mathrm{s}$, and the length of the cable is $1.3 \mathrm{~m}$. The acceleration of gravity in the local is $9.7913 \mathrm{~m} / \mathrm{s}^{2}$. An incremental bar code is installed on the girder of the crane and a scanner is installed on the trolley to measure the position of the trolley. A plotting paper is on the 
ground and a camera is installed on the trolley to measure the swing of the payload. The velocity of the trolley is precise controlled by the industrial personal computer.

Figure 6 indicates the two different input velocity of the trolley. The trolley velocity in case 1 is designed by the $\mathrm{ZV}$ input shaper. The trolley velocity in case 2 is designed by the ZVD input shaper. The input times of the two input shapers are different, however, the maximum velocity of three input shapers are same, which are about $0.084 \mathrm{~m} / \mathrm{s}$. The input amplitude and the input time of the two shaper are calculated by the input shaper parameters.

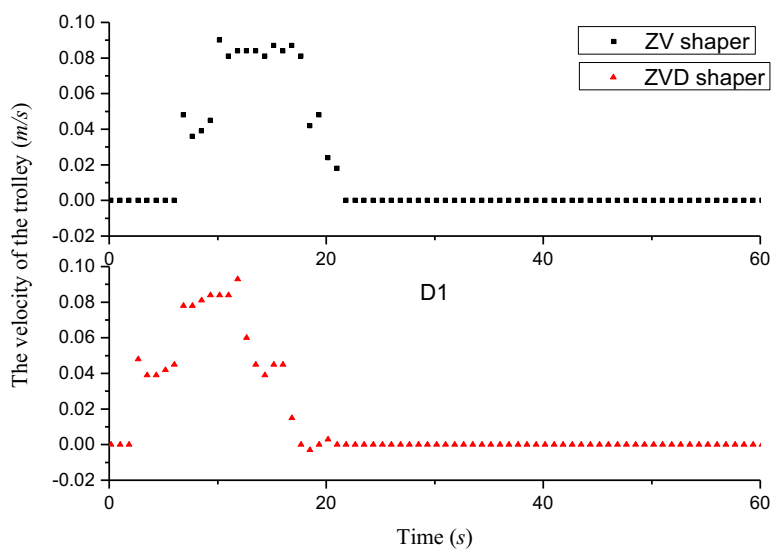

Figure 6 . the velocity of the trolley

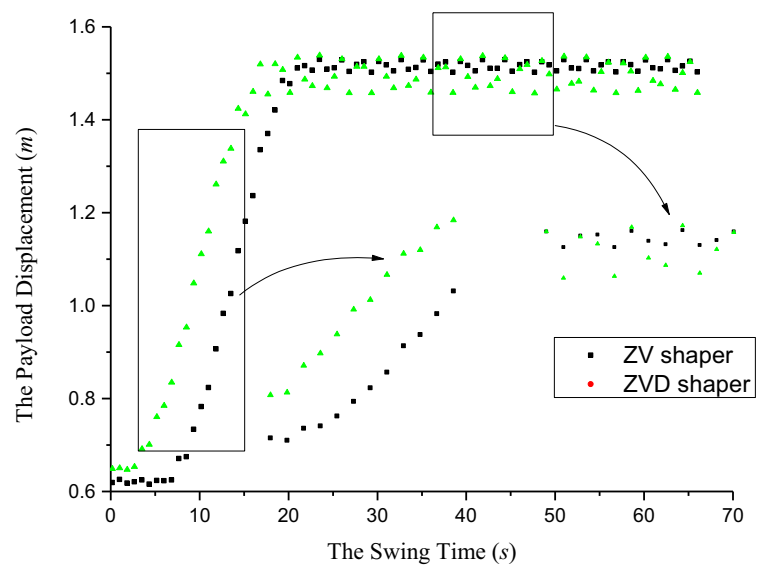

Figure 7. the displacement of the payload

The displacements of the payload when the trolley motion are shown in figure 7. The payload follows the trolley's motion while the payload swing around with the trolley at the same time. After the trolley stop moving at about 20 seconds, the payload still swings as the inertia effect. The ZVD input shaper in case 2 has the minimum swing amplitude range, and it is smoother when the trolley still moves. The residual swing amplitude of the ZV shaper is about three times bigger than ZVD shaper. The payload stopping swing time of the ZV shaper is much longer than that of ZVD input shaper. In a word, the ZVD input shaper is more effect to suppress the payload swing than the ZV input shapers.

\section{Conclusion}

A dynamic model of the overhead crane is proposed according to the physics structure of the crane. The input shaper is designed according to motion planning of the overhead crane. The mass of the trolley, the mass of payload and the length of wire rope are key factors which affect the probability distribution of the crane's swing frequency. The ZVD input shaper is better to suppress the payload residual swing when the payload moved to the expected location. The advantage of the ZVD input shaper is that the residual swing of the payload has been suppressed. The ZVD input shaper can reduce the swing time of the overhead crane when the payload move to the planning location.

\section{References}

1. E. M. Abdel-Rahman, A. H. Nayfeh, Z. N. Masoud, J. Vib. \& Ctr. 9, 863-908 (2003)

2. W. Singhose, K. L. Sorensen, S. Dickerson, Ctr. Engi. Prac. 15, 825-837 (2007)

3. M. Tong, Y. Wang, H. Qiu, Procedia Engi. 16, 342347 (2011)

4. E. M. Abdel-Rahman, A. H. Nayfeh, Pendulation reduction in boom cranes using cable length manipulation. Nonlinear Dynamics, 27, 255-269 (2002)

5. N. Sun; Y. Fang, Automatica 50, 2350-2357 (2014)

6. H. H. Lee, Y. Liang, D. Segura, J. Dyn. Sys. Meas. \& Ctr-Trans. The ASME 128, 842-845 (2006)

7. H. H. Lee, J. Dyn. Sys. Meas. \& Ctr-Trans. The ASME 126, 359-364 (2004)

8. T. Y. T. Kuo, S. C. J. Kang, Auto. Cons. 42, 25-35 (2014)

9. G. Bartolini, A. Pisano, E. Usai, Automatica 38, 1783-1790 (2002)

10. S. C. Kang, H. L. Chi, E. Miranda, J. Com. Civ. Engi. 23, 363-371(2009)

11. N. C. Singer, W. P. Seering, J. Dyn. Sys. Mea. \& Ctr. 112, 76-82 (1990) 